\title{
IMPACTO DE LAS POLÍTICAS DE ASEGURAMIENTO DE LA CALIDAD EN PROGRAMAS DE EDUCACIÓN SUPERIOR: UN ESTUDIO EXPLORATORIO
}

\section{Antecedentes}

La motivación para desarrollar la investigación cuyos primeros resultados se presentan en esta publicación surge en un escenario de incremento de la oferta educacional terciaria en Chile, en el cual se ha producido una expansión y diversificación de las instituciones de educación superior y del perfil de los estudiantes; se han incorporado nuevas tecnologías, modelos de formación por competencias y las instituciones han integrado en sus agendas la discusión acerca del perfil de sus egresados y la revisión de los planes de estudio. Estas innovaciones, que se han desarrollado progresivamente, configuran hoy una realidad instalada y forman parte del sistema de educación superior.

Dos décadas atrás, en el año 1990 se establecieron normativas y prácticas de creación y reconocimiento oficial de las instituciones de educación superior y se instituyeron mecanismos formales de información y evaluación de la calidad de los servicios para este nivel de educación. En este contexto destaca el trabajo que llevaron a cabo el Consejo Superior de Educación (CSE), la Comisión Nacional de Acreditación de Pregrado (CNAP) y la Comisión Nacional de Acreditación de Postgrado (CONAP). Con la conducción de procesos de acreditación para instituciones autónomas, que voluntariamente se sometieron a evaluación, sentaron las bases para la consolidación del actual Sistema Nacional de Aseguramiento de la Calidad de la Educación Superior, formalizado con la promulgación de la Ley 20.119 en noviembre de 2006 y que, en la actualidad, enmarca las políticas para el mejoramiento de la educación superior en Chile.

La creación de estos organismos y la puesta en marcha de la etapa experimental de procesos de acreditación de carreras e 
instituciones, impulsaron un cambio en el sistema. El desarrollo y aplicación de instrumentos y procedimientos para llevar a cabo procesos de autoevaluación y evaluación de pares externos y la experiencia de más de 450 carreras que participaron de esta etapa, movilizaron a las instituciones y las estimularon a trabajar por el mejoramiento de sus programas, estableciendo gradualmente una cultura de evaluación en un medio de creciente competencia. Es así como se puede observar que las instituciones de educación superior han incorporado en su práctica espacios de trabajo para desarrollar propuestas de mejora de sus planes de estudio y de innovación de recursos para la enseñanza, para así reclutar mejores estudiantes y optimizar los indicadores de eficiencia.

Siguiendo el razonamiento de Stensaker (2008), estos cambios, sumados a la fluidez y permeabilidad en el intercambio y difusión de información que caracteriza nuestra época, así como a la introducción de mecanismos de acreditación en un contexto de aseguramiento de la calidad, promueven que la información referida a instituciones de educación superior sea más transparente y accesible, se integre en el discurso público y posibilite que las personas interesadas tomen decisiones más informadas.

No obstante, pese a todas estas acciones e interés mostrado por representantes tanto del mundo académico como de la política pública, la evidencia acerca del impacto de estos mecanismos de aseguramiento de la calidad es aún muy escasa. No ha habido un desarrollo paralelo de estudios empíricos que investiguen acerca de la calidad de los programas y de las instituciones que los ofrecen. Los datos que se han publicado provienen del análisis de muestras muy reducidas de actores vinculados a la educación terciaria o bien de experiencias de casos únicos (Navarro, 2007; Fernández L., 2006; Rivera et al., 2009; Zurita, 2004; Monetta y Muñoz, 2004). En esta misma línea de argumentación, Lemaitre (2005) contribuye con algunas propuestas y señala que el impacto se observa en el desarrollo de recursos humanos, en aprendizajes institucionales y en la gestión institucional. Sin embargo, confirma que no existen estudios formales acerca de las consecuencias de estos mecanismos, por lo cual su reflexión y aseveraciones las hace a partir de lo que la autora 
denomina un "análisis informal" (p. 62) acerca de las consecuencias de los mecanismos de aseguramiento de la calidad y, aunque se fundamentan en una vasta experiencia, no cuentan con el sustento de datos empíricos sistematizados.

En la literatura nacional e internacional, las publicaciones acerca de la calidad de la educación superior desarrollan conceptualizaciones muy diversas (Bellingham, 2008; Lemaitre, 2007; Proyecto ALFA Nro. DCI-ALA/2008/42, 2009; Scharager et al., 2008; Stensaker, 2008; Velasco et al., 2006 entre otras), dejando en evidencia que es un constructo complejo, dinámico, multidimensional, que se suele definir por aspectos que faltan o son deficitarios más que por atributos positivos.

En medio de disparidades conceptuales, la noción de calidad que se asuma probablemente va a depender de la fuente consultada, de los marcos de referencia y los propósitos para estudiarla. Esta variedad de enfoques y aproximaciones hace difícil definir operativamente y medir el impacto de los procesos de aseguramiento de la calidad sobre los sistemas y las instituciones de educación superior, dificultando la generación de evidencia que sitúe las experiencias en un plano común de comparación.

La investigación acerca de la calidad de la educación superior basada en evidencia empírica de muestras numerosas ha estado más orientada hacia otros ámbitos. Uno de ellos es la relación entre formación de profesionales y su vínculo con el trabajo; destacan en este aspecto los estudios CHEERS (Careers After Higher Education: an European Research Survey) y REFLEX (The Flexible Professional in the Knowledge Society) (Teichler, 2002, 2007; ANECA, 2007; Allen y van der Velden, 2007). Un segundo ámbito de investigación ha sido la formación por competencias, en el cual destaca el proyecto Tuning Educational Structures in Europe, dirigido desde el ámbito universitario con el fin de ofrecer una propuesta concreta para implementar el proceso de Bolonia en las instituciones de educación superior y en diversas disciplinas y áreas de estudio de Europa y Latinoamérica (Tuning Project, 2006; Scharager y cols., 2008). Con este proceso se esperaba alcanzar el año 2010 en Europa tres metas principales: 1) 
mejorar la competitividad y atractivo internacional de la educación superior europea, 2) mejorar la empleabilidad de los graduados europeos, y 3) desarrollar la movilidad interna y externa de estudiantes y graduados (Mora, 2004).

La escasez de evidencia manifiesta la dificultad que conlleva conducir este tipo de estudios, lo que se explica por los problemas de orden conceptual mencionados, y por los inconvenientes metodológicos derivados de la compleja tarea de construir indicadores de calidad y aislar factores responsables de los cambios en las instituciones de educación superior, tal como lo afirman Stensaker (2008) y Amaral (2010).

En lo concerniente al estudio de impacto de las políticas de aseguramiento de calidad, probablemente el esfuerzo más importante es el Proyecto ALFA Nro. DCI-ALA 2008/42, denominado "Aseguramiento de la Calidad: políticas públicas y gestión universitaria”, liderado por el Centro Interuniversitario de Desarrollo, CINDA, con sede en Chile. Este proyecto, aún en curso, "involucra a 23 universidades en 12 países latinoamericanos y cuatro países europeos, tiene como objetivo general evaluar el impacto de los mecanismos de aseguramiento de la calidad en la perspectiva de políticas públicas, y desarrollar capacidades para una mejor gestión de la calidad de la educación superior"2.

A la luz de estos antecedentes, es indiscutible la necesidad de sumar esfuerzos y desarrollar investigaciones que aborden este tema e intenten dimensionar el impacto que han tenido las políticas y mecanismos de aseguramiento de la calidad en aquellos programas que han participado en procesos de acreditación. Alineado con este propósito, el estudio financiado por el Consejo Nacional de Educación (CNED), que se presenta en esta publicación, se realizó entre los meses de agosto de 2009 y abril de 2010. Los principales resultados obtenidos deben leerse con la cautela que imponen sus limitaciones, particularmente en lo referente a sus aspectos metodológicos, que se explican más adelante.

2 Se puede acceder a los antecedentes de este proyecto en http://www.cinda.cl/proyecto_alfa/ $\mathrm{htm} /$ documentos.htm 
Este estudio de carácter exploratorio se diseñó con el fin de contrastar algunas hipótesis. Se esperaba encontrar diferencias en las percepciones de impacto en sus instituciones producto de la participación en procesos de acreditación; como asimismo, entre grupos de carreras clasificadas en cuatro categorías operacionales, que jerarquizan las instituciones participantes en torno a un continuo definido por el tiempo de acreditación (años) y la cantidad de carreras sometidas a evaluación por institución. Con la finalidad de hacer operativa la recolección de datos de percepciones de impacto, fue necesario acotar la conceptualización subyacente a la evaluación, en un contexto en el que no se dispone de una definición consensuada del significado de impacto de las acciones orientadas al aseguramiento de la calidad de la educación superior, ni de indicadores probados. De este modo, aplicando un término que utiliza Stensaker (2008), en este estudio en lugar de medir directamente el impacto mediante de indicadores objetivos, lo que se hizo fue indagar y dimensionar los efectos de los mecanismos de promoción de calidad en la educación superior, a través de datos que constituyen "cuasi-indicadores" de impacto. Estos los conforman los registros de las percepciones y juicios de los informantes acerca de los cambios experimentados en sus organizaciones y programas después de haber realizado un proceso de acreditación con la CNAP.

Para estos efectos, se administró una encuesta en línea que se envió a las 460 carreras acreditadas entre 2001 y 2006. Se recibió respuesta de 95 carreras, lo que representa un 20\% del universo. Los resultados confirmaron parcialmente las hipótesis, identificando menos diferencias entre las carreras de lo esperado. Aquellas que pertenecen a instituciones con un mayor número de carreras acreditadas y por más años en el periodo en estudio, tienden a dar cuenta de menos cambios en las condiciones de operación que aquellas que pertenecen a instituciones con un número menor de carreras acreditadas y por menos años. Este resultado probablemente se deba al hecho de que sus condiciones preexistentes eran de mejor nivel y más consolidadas antes de la acreditación, lo que explica que se percibieron menores efectos de los procesos de acreditación. 


\section{Objetivos}

Los objetivos fueron llevar a cabo un estudio de carácter exploratorio que permitiera (1) construir un registro del juicio que hacen los directores de unidades académicas y encargados de aseguramiento de la calidad en cada institución, acerca del impacto percibido en sus organizaciones, como consecuencia de la participación de las carreras en procesos de acreditación y (2) comparar perfiles de impacto según la categoría de las carreras, en función de las dimensiones de evaluación para la acreditación en Chile.

\section{Marco conceptual}

La evaluación de un programa tiene como finalidad: 1) juzgar si se han alcanzado los objetivos y 2) determinar si las innovaciones introducidas han tenido algún impacto. Un sistema de evaluación trata de pronunciarse acerca del valor de un programa con el fin de tomar decisiones sobre su ejecución. Por lo tanto, a partir de sus resultados se puede establecer si el programa debe seguir siendo implementado, debe eliminarse o debe experimentar modificaciones (Fernández-Ballesteros, 1996).

Con relación al concepto de calidad, Fernández Lamarra (2007) señala que éste no es un concepto unívoco y fijo sino que se construye a través de consensos y negociaciones entre los actores. De esta manera, para los académicos se refiere principalmente a los saberes, para los empleadores a competencias, para los estudiantes a la empleabilidad, para la sociedad a ciudadanos respetables y capaces, para el Estado, según la concepción que asuma, puede variar de aspectos vinculados con el desarrollo social y humano a la eficiencia, costos y requerimientos de capital humano.

Para los fines de este estudio, se ha optado por un enfoque de evaluación analítica, que distingue los componentes que se indican en la tabla 1 y se describen a continuación. 


\section{Componentes de la evaluación}

La determinación de componentes de evaluación se ha hecho sobre la base de distinciones que provienen de dos ámbitos. Por un lado, se han tomado como referencia las conceptualizaciones de evaluación de programas (Stufflebeam \& Shinkfield, 1985; Briones 1985), que reconoce la estructura, funcionamiento y resultados de un programa, con la finalidad de proporcionar información de la cual se deriven criterios útiles para la toma de decisiones en relación con su administración y desarrollo; y, por otro lado, los criterios de acreditación nacional de programas de educación superior (CNAP, 2002; CNA, 2009). Los componentes son:

\section{Estructura}

Se refiere al modo en el que el programa o carrera articula sus recursos materiales y humanos ${ }^{3}$ en actividades dirigidas al logro de los objetivos, inserto en una institución de educación superior a la que pertenece. Incluye aspectos como coherencia con los propósitos institucionales; condiciones normativas (reglamentos, sistemas de información, mecanismos de retroalimentación, entre otros.) y estructura organizacional académico-administrativa del programa. Los factores incluidos en este componente contienen principalmente elementos de las dimensiones Condiciones de Operación y Autorregulación, de acuerdo con la clasificación de criterios de acreditación de carreras que aplicaba la CNAP.

\section{Proceso de formación}

Se refiere a aspectos como organización curricular y métodos pedagógicos; dotación académica; mecanismos de apoyo a los estudiantes, calidad y cantidad de infraestructura, apoyo técnico y recursos para la enseñanza; todos estos, elementos necesarios para satisfacer plenamente los propósitos, y que, en general se superponen con las dimensiones de Perfil de Egreso y Condiciones de Operación.

3 Los recursos humanos, en el contexto de esta dimensión, se refieren principalmente a aquellos que están a cargo de funciones directivas, académicas y administrativas. 


\section{Resultados}

Se refiere a los productos (percepción de la calidad de la formación de egresados) y cumplimiento de los propósitos de la carrera, tomando en consideración los vínculos con el medio local y disciplinario y las características del perfil de egreso de los profesionales. Asimismo, se incluyen aspectos de la organización que experimentan transformaciones, a partir de las acciones de aseguramiento de calidad. Estos comprenden aquellos ámbitos institucionales, como son los sistemas de gobierno, control y autorregulación, estructuras, procedimientos y elementos de la cultura institucional. Los factores que se evalúan en este componente se encuentran en las tres dimensiones de criterios de evaluación para la acreditación de carreras.

Con el objeto de integrar estas categorías de análisis con las dimensiones empleadas en el sistema de evaluación para la acreditación en Chile, se construyó una matriz que los combina (criterios aplicados en la acreditación y componentes de la evaluación, según los autores citados), como se muestra en la tabla 1.

De esta manera, el componente Estructura se relaciona con los criterios CNAP incluidos en las dimensiones Condiciones de Operación y Capacidad de Autorregulación; el componente Proceso de Formación con los criterios de Perfil de Egreso y Resultados, y Condiciones de Operación; y el componente Resultados e Impacto con las tres dimensiones. Para efectos de análisis y presentación de resultados, se hará referencia a las distinciones de criterios utilizados en el ámbito de la acreditación de carreras, precisando el componente de la matriz de evaluación de programas (estructura, procesos y resultados).

\section{Población}

La población objetivo de este estudio puede considerarse como una muestra sesgada de la totalidad de instituciones de educación superior del país, ya que conforman el universo de aquellas que decidieron voluntariamente presentarse a una evaluación de pares, luego de un proceso de autoevaluación, con el fin de obtener una acreditación, 
que en esos años (2001-2006) no estaba regulada por ninguna norma o ley y no conducía a beneficios o incentivos adicionales. Esta particular naturaleza de la población impone una limitación metodológica que reduce la posibilidad de generalizar los resultados a programas que no participaron de procesos de acreditación en ese periodo, condición que restringe la transferencia de los hallazgos sólo a aquellas unidades académicas que pertenecen a este universo o comparten sus características.

Tabla 1. Dimensiones de análisis utilizadas en estudio del impacto de la participación en procesos de acreditación durante el periodo 2001-2006 conducidos por CNAP.

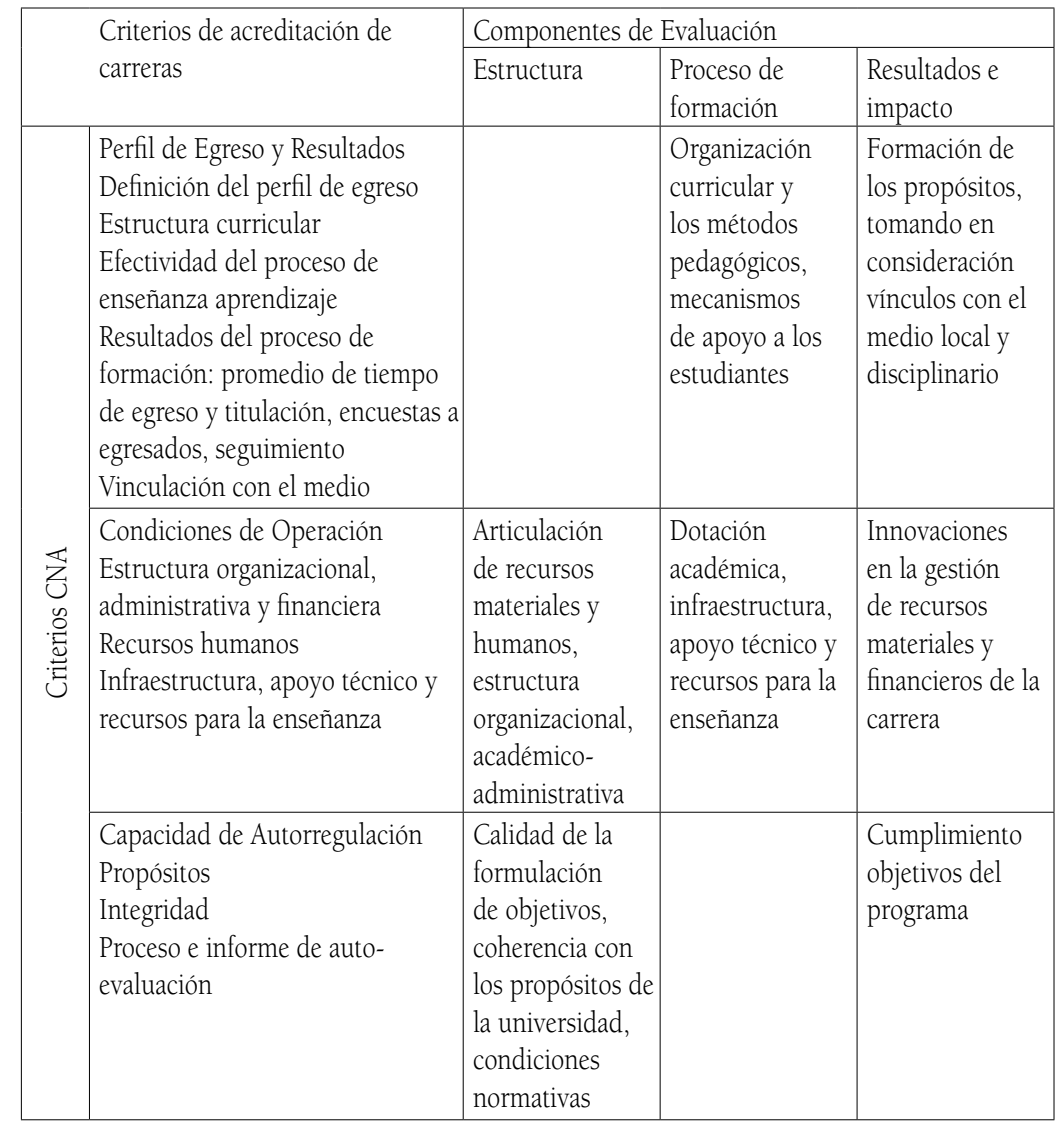

El marco muestral con el total de carreras se construyó a partir de una base de datos de los registros de aquellas que participaron en procesos de acreditación conducidos por la CNAP entre los años 2001 
y 2006. En el mes de agosto de 2009 se extrajeron los antecedentes disponibles en la página web www.cnachile.cl, que contenía esta información (nombre de la institución, área de la carrera, carrera, sede, jornada, años de acreditación de la carrera, acreditación institucional). Adicionalmente, para efectos de contacto y posterior seguimiento de las respuestas al cuestionario, se agregaron variables de identificación de personas involucradas en esos procesos.

Con el fin de asegurar cierta representatividad de la muestra de participantes, fue necesario definir un procedimiento para generar distinciones entre las carreras, que reflejaran un grado de diversidad y permitiera jerarquizarlas, de acuerdo al objetivo del estudio. Para esto, se construyó un sistema de categorías a partir de la combinación de dos criterios: (1) número promedio de años de acreditación por carrera y (2) número de carreras acreditadas por institución. Esta clasificación permite diferenciar cuatro grupos de instituciones de educación superior que se ordenan en torno a un continuo definido por el tiempo de acreditación (años) y la cantidad de carreras sometidas a evaluación por institución en el periodo de estudio. Las cuatro categorías generadas de acuerdo a este mecanismo, y la distribución porcentual de las carreras, se observan en la tabla 2. Las instituciones incluidas en la primera categoría son aquellas con menos años de acreditación (considerando a las que no fueron acreditadas) y pertenecen a instituciones que presentaron menor número de programas acreditados; mientras que las de la cuarta categoría pertenecen a instituciones que acreditaron en promedio 22 programas, por lo cual son las que acreditaron más carreras y por más tiempo (más de cuatro años).

\section{Muestra}

La muestra se obtuvo por un procedimiento no probabilístico. Se invitó a directivos de los 460 programas pertenecientes a 39 instituciones de educación superior a través del país que conformaban el universo de estudio. Se determinó una cuota de $20 \%$ como tasa mínima de respuestas por categoría. Se implementó un sistema de seguimiento motivacional mediante correos electrónicos y comunicación telefónica, para que los participantes respondieran, con esfuerzos selectivos 
privilegiando aquellas categorías de carreras con baja respuesta, para asegurar el cumplimiento de la proporción prefijada. Finalmente, el total de respuestas obtenidas fue de 95 (tasa de respuesta final: 20\% (ver tabla 2).

Tabla 2. Distribución de instituciones y carreras participantes del estudio, según muestreo por cuotas para cada categoría de IES (nivel de participación y resultado de acreditación).

\begin{tabular}{|c|c|c|c|c|c|}
\hline $\begin{array}{c}\text { Categoría } \\
\text { IES }\end{array}$ & $\begin{array}{c}\text { N. }{ }^{\circ} \text { total IES } \\
\text { por categoría }\end{array}$ & $\begin{array}{c}\text { N. }{ }^{\circ} \text { de carreras } \\
\text { por categoria }\end{array}$ & $\begin{array}{c}\text { Cuota }(20 \%) \\
\text { esperada de carrera } \\
\text { por categoría }\end{array}$ & $\begin{array}{c}\text { N. }{ }^{\circ} \text { carreras de } \\
\text { la muestra por } \\
\text { categoría }\end{array}$ & $\begin{array}{c}\% \text { respuestas } \\
\text { por categoría }\end{array}$ \\
\hline 1 & 9 & 59 & 12 & 18 & $31 \%$ \\
\hline 2 & 12 & 72 & 14 & 15 & $21 \%$ \\
\hline 3 & 8 & 100 & 20 & 25 & $25 \%$ \\
\hline 4 & 8 & 228 & 46 & 37 & $16 \%$ \\
\hline TOTAL & $37^{*}$ & 459 & 92 & 95 & $20,7 \%$ \\
\hline
\end{tabular}

* Nota: no se incluyó la Escuela Militar del Libertador General Bdo. O’Higgins porque sólo tenía una carrera.

\section{Instrumento de recolección de datos}

El cuestionario que se envió a los participantes se compone de preguntas abiertas y cerradas (escalas de tipo Likert y de alternativas de respuesta dicotómicas), dirigidas a recabar opiniones de directores de carreras y encargados institucionales de aseguramiento de la calidad en el periodo próximo a la acreditación. Este fue elaborado especialmente para el estudio y está orientado a caracterizar las percepciones de cambios producidos en las unidades académicas, de acuerdo a una comparación temporal antes/después del proceso de acreditación en sus carreras, como consecuencia de la participación en estos procesos, en el periodo 2001-2006. Este instrumento se estructuró en cuatro secciones, las tres primeras abordan un espacio o componente en la organización en el que se produce el cambio, identificando respectivamente el ámbito de ocurrencia, la importancia atribuida a su ocurrencia y el nivel de la organización en que se localiza la gestión del cambio, y la cuarta sección identifica y dimensiona la estabilidad de resultados atribuibles a la participación en acreditación de carreras (tabla 3). 
Tabla 3. Secciones del cuestionario para evaluar percepción de cambios atribuibles a la participación en procesos de acreditación periodo 2001-2006.

I. Identificación de cambios percibidos en las carreras en procesos o estamentos. Presenta un listado de 40 planteamientos que se formulan en forma de pregunta:

¿En mi carrera se han realizado cambios, ajustes o innovaciones en...?

II. Grado de importancia atribuida a los cambios producidos en cada uno de estos elementos (responde a la pregunta presenta el mismo listado de 40 planteamientos en forma de pregunta: ¿Qué grado de importancia le atribuye usted a estos cambios, ajustes o innovaciones realizadas en su carrera?).

III. Localización de la entidad responsable de estos cambios. Presenta el mismo listado de 40 planteamientos y solicita dar respuesta a la pregunta: ¿La administración de estos cambios es responsabilidad del nivel central de la institución o son responsabilidad de la carrera?

IV. Resultados producidos como consecuencia de la acreditación. Presenta un listado de 25 cambios posibles en distintas dimensiones y pide que se responda a la pregunta: para cada uno de ellos, señale si ha disminuido, se ha mantenido o ha aumentado desde el año 2001 a la fecha.

\section{Construcción del cuestionario}

La construcción del instrumento se hizo en dos etapas. En la primera se elaboró un cuestionario preliminar, con preguntas abiertas, con el propósito de (1) conocer la opinión de representantes de universidades acerca de la noción de calidad de educación superior que se maneja en la cotidianeidad del trabajo académico y (2) obtener categorías de indicadores de impacto de procesos de acreditación, de acuerdo a la experiencia de los propios actores involucrados. Este primer cuestionario constituyó la pauta para la construcción del instrumento definitivo. Como participantes de esta etapa se invitó a responder a representantes de cuatro instituciones de educación superior que tenían las siguientes características:

- Institución pública del área metropolitana

- Institución privada del área metropolitana

- Institución pública regional

- Institución privada regional

Los invitados a participar respondieron aportando información relevante, la que se analizó cualitativamente y ayudó a orientar la creación de categorías de respuestas, agrupadas de acuerdo a los criterios de acreditación de carreras CNAP y los componentes de evaluación, conforme a la matriz de la tabla 1 . Los resultados de 
la aplicación de este primer cuestionario sirvieron de base para la construcción del instrumento definitivo, en la segunda etapa.

\section{Subida de los instrumentos al sitio web y aplicación}

El cuestionario definitivo se localizó en un sitio web, de fácil acceso para los encuestados, para ser respondido en línea. Se envió una invitación a los destinatarios y en los dos meses siguientes se reiteró -tanto vía correo electrónico como telefónica- la invitación. Las tasas de respuestas, respecto de la muestra final, fueron las siguientes: $17 \%$, en diciembre $2009,62 \%$ en enero, $2 \%$ en febrero y $19 \%$ en marzo de 2010. Luego del terremoto ocurrido en Chile, el 27 de febrero, se decidió no insistir en aquellas carreras ubicadas en universidades de las regiones VII y VIII (las más dañadas por el sismo). Al cabo de ocho reiteraciones en las carreras ubicadas en el resto del país, se suspendió la recolección de datos la primera semana de abril al constatar que la tasa de respuesta después de mediados de marzo no había aumentado, pese al seguimiento telefónico y electrónico, lográndose una cuota global de $20 \%$ y cumpliendo con la cuota fijada inicialmente para tres de las cuatro categorías (en la categoría 4, se obtuvo sólo un 16\% de respuestas), como se muestra en la tabla 2.

\section{Resultados}

\section{Cambios identificados}

La mayor proporción de respuestas de cambios considerados por los participantes como un efecto de los procesos de acreditación de sus carreras, se concentra en aquellas que fueron clasificadas en las tres primeras categorías ${ }^{4}$ Las carreras de la categoría cuatro, aunque reconocen cambios, lo hacen en una proporción menor de acuerdo a los indicadores contenidos en los reactivos del cuestionario. La dimensión en la que se expresa mejor esta diferencia entre las carreras de instituciones de la categoría cuatro y las otras, es la Capacidad

4 La categoría 1 indica menor número de programas ingresados a acreditación y años de acreditación inferior a tres, en comparación con aquellas carreras que pertenecen a instituciones que acreditaron en promedio 22 programas y obtuvieron más de cuatro años de acreditación y se clasificaron en la categoría cuatro. 
de Autorregulación. Las dos innovaciones más reportadas en esta dimensión, las que a su vez se ubican dentro del componente Procesos de Formación (ver tabla 1), son la implementación de un sistema de información para orientar el proceso de autoevaluación para la acreditación y creación de una cultura de evaluación. En ambas, aunque son reconocidas como indicadores de cambio por un porcentaje importante de las carreras de la cuarta categoría, en promedio, suman una proporción menor que las otras tres (ver tabla 4).

Tabla 4. Distribución porcentual de carreras por categoría con cambios en sus unidades asociados a procesos de acreditación en la dimensión Capacidad de Autorregulación

\begin{tabular}{|l|c|c|c|c|c|}
\hline FACTORES DIMENSIÓN AUTORREGULACIÓN & Cat. 1 & Cat. 2 & Cat. 3 & Cat. 4 & Promedio \\
\hline Definición de políticas de docencia & $59 \%$ & $44 \%$ & $56 \%$ & $54 \%$ & $53 \%$ \\
\hline $\begin{array}{l}\text { Formalización de procesos a través de manuales o } \\
\text { guías de procedimientos }\end{array}$ & $76 \%$ & $50 \%$ & $52 \%$ & $46 \%$ & $56 \%$ \\
\hline Actualización de reglamentos generales & $88 \%$ & $38 \%$ & $72 \%$ & $62 \%$ & $65 \%$ \\
\hline $\begin{array}{l}\text { Mecanismos de difusión de reglamentos de carrera } \\
\text { en los distintos estamentos }\end{array}$ & $65 \%$ & $69 \%$ & $76 \%$ & $68 \%$ & $69 \%$ \\
\hline $\begin{array}{l}\text { Diseño de un sistema de información para orientar } \\
\text { el proceso de autoevaluación para la acreditación }\end{array}$ & $94 \%$ & $81 \%$ & $80 \%$ & $62 \%$ & $79 \%$ \\
\hline Cultura de evaluación & $94 \%$ & $81 \%$ & $80 \%$ & $70 \%$ & $81 \%$ \\
\hline $\begin{array}{l}\text { Implementación de un sistema de información } \\
\text { para orientar el proceso de autoevaluación para la } \\
\text { acreditación }\end{array}$ & $94 \%$ & $88 \%$ & $84 \%$ & $70 \%$ & $84 \%$ \\
\hline PROMEDIO & $83,1 \%$ & $66,4 \%$ & $73,0 \%$ & $61,8 \%$ & \\
\hline
\end{tabular}

La dimensión Condiciones de Operación es la que presenta la mayor dispersión de respuestas; éstas se distribuyen en un rango de variación promedio que contiene desde un grupo minoritario (18\%) que señala la creación de cargos de asistente administrativo para la gestión de temas estudiantiles hasta el grupo más numeroso (73\%) que consigna la implementación de sistemas de información (registros académicos) de apoyo a la docencia, como cambio asociado a los procesos de acreditación. En ambos casos, la tendencia es muy homogénea entre las cuatro categorías. Otros elementos en los que se manifiestan cambios de frecuente nominación son las innovaciones en recursos de apoyo a la docencia y la creación de comités curriculares. En éstos no hay diferencias importantes entre categorías. Por otro lado, hay un conjunto de cambios que son señalados mayoritariamente por las carreras de las categorías primera a tercera. Los más nombrados son: 
- introducción de consejos asesores,

- desarrollo de instrumentos para evaluar el desempeño docente,

- desarrollo de criterios de asignación de recursos destinados a renovar o mejorar la dotación de infraestructura e

- instalaciones y recursos para la docencia y para la investigación.

Probablemente en las carreras de la categoría 4, éstas eran condiciones preexistentes, de un buen nivel de calidad y adecuadas a los requerimientos; por lo tanto, no se ven impactadas por los procesos de evaluación externa, como sí ocurre en las carreras de las tres primeras categorías (ver tabla 5).

Tabla 5. Porcentaje de carreras por categoría que indican cambios en sus unidades asociados a procesos de acreditación en los factores con más menciones: dimensión Condiciones de Operación

\begin{tabular}{|l|c|c|c|c|c|}
\hline $\begin{array}{l}\text { FACTORES DIMENSIÓN CONDICIONES DE } \\
\text { OPERACIÓN CON MAYORES DENOMINACIONES }\end{array}$ & Cat. 1 & Cat. 2 & Cat. 3 & Cat. 4 & Promedio \\
\hline Comités curriculares & $65 \%$ & $69 \%$ & $76 \%$ & $65 \%$ & $69 \%$ \\
\hline Consejos asesores & $82 \%$ & $69 \%$ & $72 \%$ & $46 \%$ & $67 \%$ \\
\hline $\begin{array}{l}\text { Desarrollo de instrumentos para evaluar el } \\
\text { desempeño docente }\end{array}$ & $82 \%$ & $44 \%$ & $76 \%$ & $54 \%$ & $64 \%$ \\
\hline $\begin{array}{l}\text { Sistemas de información (registros académicos) de } \\
\text { apoyo a la docencia }\end{array}$ & $88 \%$ & $75 \%$ & $68 \%$ & $62 \%$ & $73 \%$ \\
\hline Recursos de apoyo a la docencia & $88 \%$ & $69 \%$ & $68 \%$ & $62 \%$ & $62 \%$ \\
\hline Mecanismos para seguimiento y apoyo a la gestión & $65 \%$ & $75 \%$ & $60 \%$ & $43 \%$ & $61 \%$ \\
\hline $\begin{array}{l}\text { Desarrollo de criterios de asignación de recursos } \\
\text { destinados a renovar o mejorar la dotación de } \\
\text { infraestructura, instalaciones y recursos para la } \\
\text { docencia }\end{array}$ & $71 \%$ & $56 \%$ & $60 \%$ & $41 \%$ & $57 \%$ \\
\hline $\begin{array}{l}\text { Desarrollo de criterios de asignación de recursos } \\
\text { destinados a renovar o mejorar la dotación de } \\
\text { infraestructura, instalaciones y recursos para la } \\
\text { investigación }\end{array}$ & $71 \%$ & $63 \%$ & $40 \%$ & $41 \%$ & $53 \%$ \\
\hline PROMEDIO & $77 \%$ & $65 \%$ & $65 \%$ & $52 \%$ & \\
\hline
\end{tabular}

Algunos factores puntuales en los que se detectan diferencias importantes entre las categorías de carreras, son aquellos que tienen relación con la formalización de algunos procesos, como son los manuales o guías de procedimientos y el desarrollo de instrumentos para evaluar desempeño docente, en los que una mayor proporción de carreras de la categoría 1 informa de cambios generados a raíz de la experiencia de acreditación (ver gráficos 1 y 2). Como se verá 
más adelante, esta diferencia se asocia también con el nivel de la organización que se hace cargo de la gestión de estos procesos, que en el caso de las carreras de la primera categoría se dan mayoritariamente en el nivel de gobierno central de la institución. Estos datos podrían ser indicador de menor madurez o solidez organizacional o estar determinados por la existencia de diferentes estilos o culturas de gestión que se expresa en menores grados de autonomía de estas carreras, tal como lo sugieren las referencias del estudio de las estructuras de gestión de las universidades chilenas que pertenecen al Consejo de Rectores (Atria, 2005).

Gráfico 1. Desarrollo de instrumentos para evaluar desempeño docente según Categoría IES $(\mathrm{F}=13,466 \mathrm{p}=0,036)$

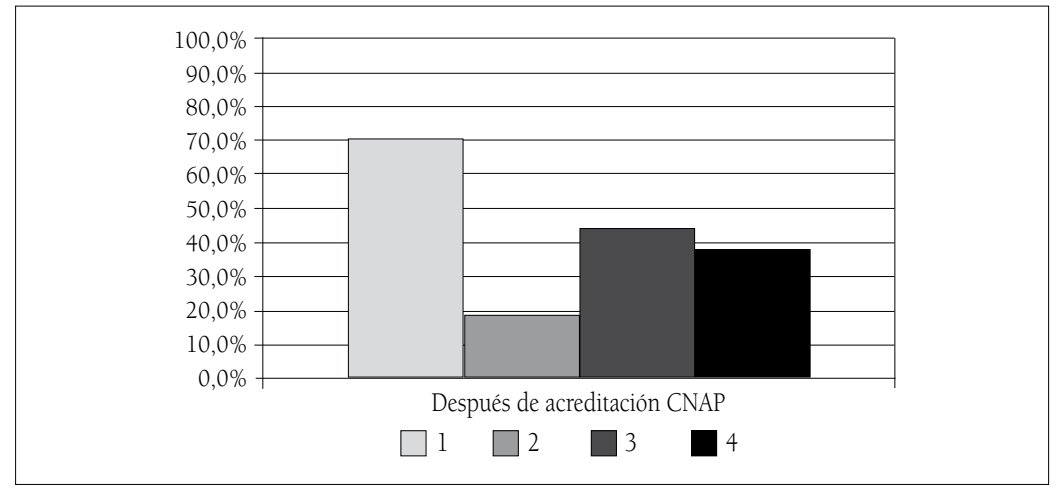

Gráfico 2. Formalización de procesos a través de manuales o guías de procedimientos según Categoría IES

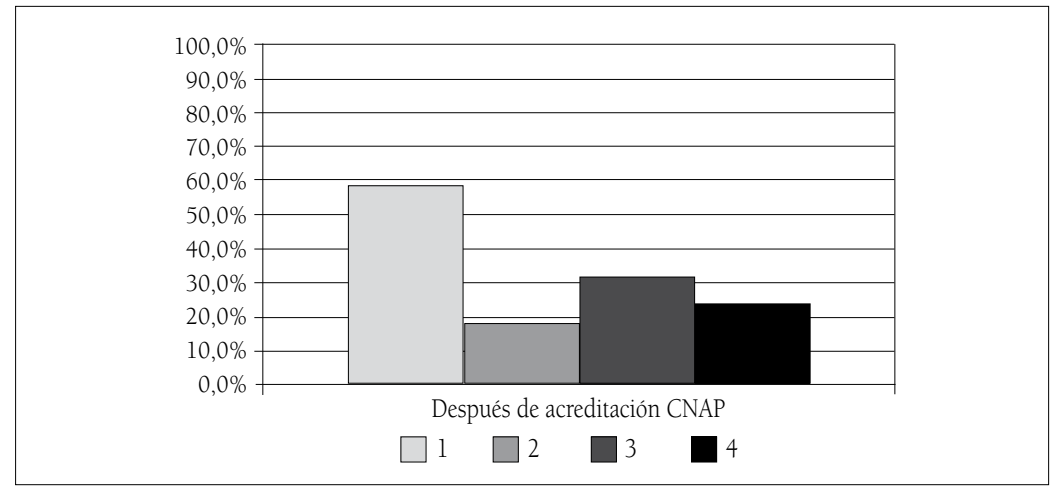




\section{Valoración de los cambios}

Por otra parte, independiente que hubieran experimentado cambios en distintas proporciones y medidas en todos los factores de análisis, las carreras de las cuatro categorías tienden a valorar los ajustes o innovaciones en estos elementos en un nivel de importancia bastante homogéneo. Así por ejemplo, al examinar la dimensión Perfil de Egreso y Resultados, todas las carreras coinciden en otorgar la valoración más baja a un mismo factor (desarrollo de estrategias de difusión y marketing), y le asignan un grado de importancia bastante parejo al resto de factores de la dimensión. Los factores que promedian más alto en las puntuaciones de importancia, son los mecanismos de apoyo a los estudiantes y aquellos destinados al seguimiento y apoyo a los procesos formativos. Esta tendencia se ilustra en los gráficos 3 y 4.

Con la intención de explorar otras asociaciones, se estableció una jerarquización de las carreras de acuerdo al número de años de acreditación obtenidos, independiente de la cantidad de otras carreras de la misma institución que se hubieran acreditado voluntariamente en el mismo periodo. A partir de esta comparación, se observa que, si bien todas las carreras consideran que los cambios producidos en la dimensión Perfil de Egreso son importantes, las carreras con más alta acreditación (6-7 años) le asignan menor importancia que aquellas con nivel de acreditación bajo y medio.

Gráfico 3. Importancia asignada a los mecanismos de apoyo a los estudiantes según Categoría IES

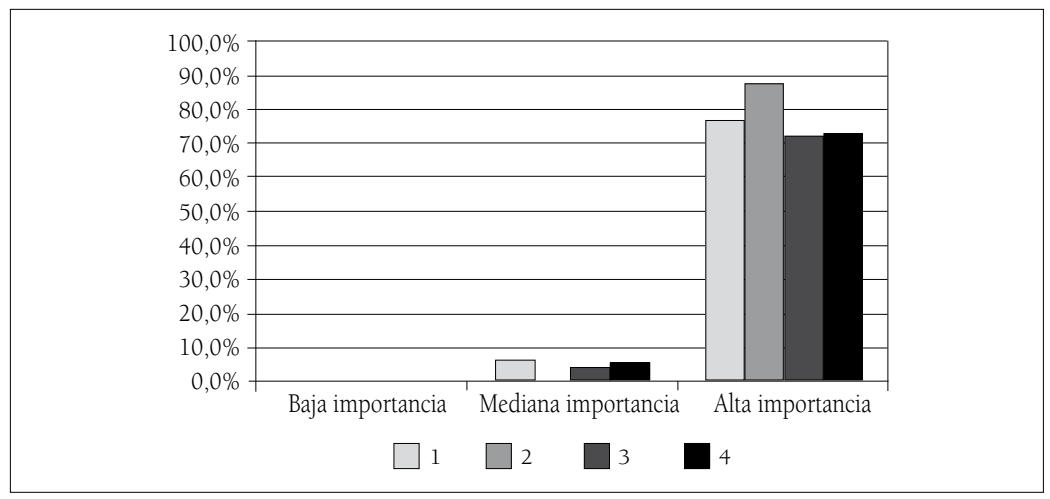


Gráfico 4. Importancia asignada a registros de seguimiento de planes de estudio según Categoría IES

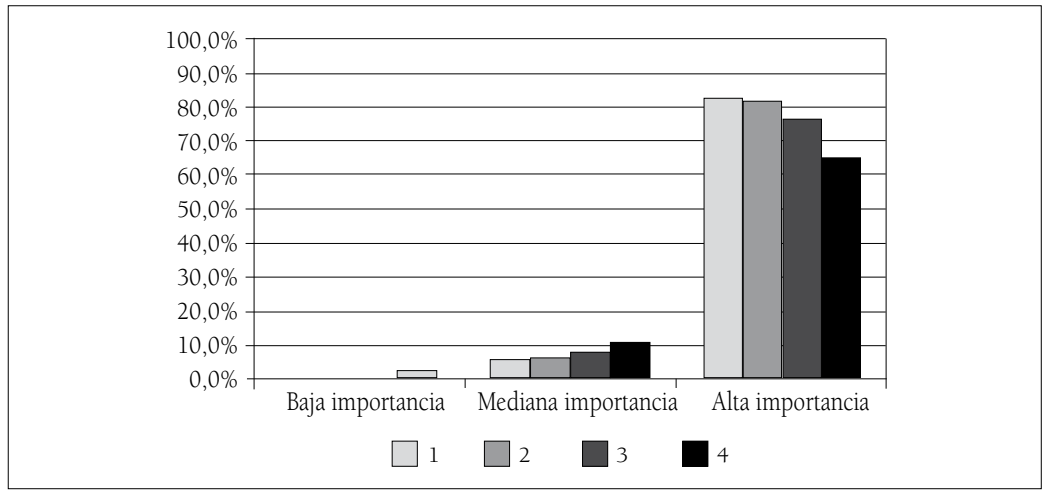

Lo mismo ocurre con la importancia que se otorga a los cambios producidos en la dimensión Condiciones de Operación y a los cambios observables en la Capacidad de Autorregulación, donde las carreras con bajo nivel de acreditación (2-3 años) tienden a asignarle una mayor importancia a los cambios que aquellas con nivel medio y alto.

Dentro del cuestionario, también se indagó a través de una pregunta abierta cuáles eran los tres cambios más importantes realizados en su carrera que pudieran atribuirse de una u otra forma al proceso de acreditación conducido por la CNAP. El 18\% de las respuestas señaló que uno de los más importantes era la generación de una cultura de evaluación al interior de las carreras (18\% categoría $1 ; 20 \%$ categoría $2 ; 20 \%$ categoría 3 y 16\% categoría 4$)$. Un segundo cambio que destacaron es la actualización del plan de estudios, éste es de mayor importancia para las carreras de la categoría 4, quienes lo señalan en un $21 \%$ de los casos, mientras que las carreras de categoría 2 sólo lo hacen en un $2 \%$. Un tercer cambio constatado por los actores consultados es el aumento en la vinculación con el medio, lo que fue mencionado por un $7 \%$ de los participantes, no presentando diferencias entre las categorías. La tabla 6 muestra la distribución de respuestas entre categorías de las menciones más frecuentes.

Al comparar la percepción de cambios entre carreras con distinto número de años de acreditación, no se observan diferencias significativas en ninguna dimensión. Sólo se advierte que se reproduce una tendencia parecida a la observada en la distinción por categoría 
institucional, en el sentido de que las carreras con mayor nivel de acreditación tienden a percibir un menor número de cambios atribuibles a los procesos de acreditación. Pero esta tendencia no marca diferencias estadísticamente significativas.

Tabla 6. Distribución de respuestas entre categorías de los tres cambios valorados como de mayor importancia: menciones más frecuentes (pregunta abierta).

\begin{tabular}{|l|l|c|c|c|c|c|}
\hline Cambios valorados como más importantes & & \multicolumn{5}{|c|}{ Categoría } \\
\hline & & 1 & 2 & 3 & 4 & Total \\
\hline \multirow{2}{*}{ Seguimiento de egresados } & $\mathrm{N} .^{\circ}$ & 3 & 1 & 4 & 3 & 11 \\
\cline { 2 - 8 } & $\%$ del total & $1 \%$ & $0 \%$ & $2 \%$ & $1 \%$ & $5 \%$ \\
\hline \multirow{2}{*}{ Revisión perfil de egreso } & $\mathrm{N} .^{\circ}$ & 1 & 0 & 3 & 7 & 11 \\
\cline { 2 - 8 } & $\%$ del total & $0 \%$ & $0 \%$ & $1 \%$ & $3 \%$ & $5 \%$ \\
\hline \multirow{2}{*}{ Cultura de autoevaluación } & $\mathrm{N} .^{\circ}$ & 7 & 8 & 13 & 15 & 43 \\
\cline { 2 - 8 } & $\%$ del total & $3 \%$ & $3 \%$ & $5 \%$ & $6 \%$ & $18 \%$ \\
\hline \multirow{3}{*}{ Aumento en vinculación con el medio } & $\mathrm{N} .^{\circ}$ & 2 & 2 & 5 & 7 & 16 \\
\cline { 2 - 8 } & $\%$ del total & $1 \%$ & $1 \%$ & $2 \%$ & $3 \%$ & $7 \%$ \\
\hline Aseguramiento de la calidad & $\mathrm{N} .^{\circ}$ & 1 & 6 & 3 & 3 & 13 \\
\cline { 2 - 7 } & $\%$ del total & $0 \%$ & $3 \%$ & $1 \%$ & $1 \%$ & $5 \%$ \\
\hline \multirow{2}{*}{ Actualización plan de estudios } & $\mathrm{N} .{ }^{\circ}$ & 6 & 1 & 11 & 19 & 37 \\
\cline { 2 - 7 } & $\%$ del total & $3 \%$ & $0 \%$ & $5 \%$ & $8 \%$ & $16 \%$ \\
\hline Total & $\mathrm{N} .^{\circ}$ & 40 & 41 & 65 & 92 & 238 \\
\cline { 2 - 7 } & $\%$ del total & $17 \%$ & $17 \%$ & $27 \%$ & $39 \%$ & $100 \%$ \\
\hline
\end{tabular}

\section{Administración del cambio}

Se preguntó a los participantes si la administración de los cambios producidos luego de la acreditación con la CNAP era responsabilidad del nivel central de la institución o de la carrera. Resultó que todas, salvo las de la categoría 1 , perciben que los cambios en la dimensión Perfil de Egreso son principalmente (60,9\%) responsabilidad de la carrera; mientras que aquellas de la categoría 1 consideran que son responsabilidad tanto del nivel central como de la carrera. Los factores de esta dimensión se concentran mayoritariamente en el componente de Procesos de Formación en la matriz de evaluación. Hay sólo dos factores que las carreras, independiente de su categoría, consideran que son casi de exclusiva responsabilidad de la unidad; éstos son la revisión de perfiles de egreso $(93,2 \%)$ y el rediseño de plan de estudios $(87,3 \%)$. 
Al repetir el análisis, comparando los niveles de acreditación de las carreras en estudio (número de años de acreditación) se observa que, independiente de los años obtenidos, las unidades consideran que los cambios producidos en la dimensión Perfil de Egreso son, en su mayoría (58,8\%), responsabilidad de la carrera. Al igual que en el caso anterior, la revisión de perfiles de egreso y el rediseño de plan de estudios son considerados como de casi exclusiva responsabilidad de la carrera (89,6\% y 85,9\%, respectivamente). Este resultado no sorprende, ya que son precisamente estas acciones las que requieren de la reflexión específica de cada disciplina y no basta con una buena capacidad de gestión académica para llevarlas a cabo con éxito, aunque la estructura de gestión sea altamente centralizada en la institución.

En la dimensión Condiciones de Operación, cuyos reactivos en el cuestionario corresponden mayoritariamente al componente Estructura según la matriz de evaluación, se observa que los cambios producidos son considerados de responsabilidad del nivel central, siendo las carreras de la categoría 1 las que le atribuyen una mayor responsabilidad $(80,3 \%)$ que las de la categoría 4 (49,3\%); estas diferencias son estadísticamente significativas $(\mathrm{F}=5,342 \mathrm{p}<0,005)$. Asimismo, se identificaron cambios que, independiente de la categoría, las carreras consideran de casi exclusiva responsabilidad del nivel central; entre ellos destacan: sistemas de información de apoyo a la docencia (registros académicos) (80,3\%), sistema de calificación y jerarquización docente (81\%), desarrollo de criterios de asignación de recursos destinados a renovar o mejorar la dotación de infraestructura, instalaciones y recursos para la docencia $(83,9 \%)$ y patrimonio e inversiones $(84,4 \%)$.

Nuevamente, al reproducir el análisis de estos datos con relación al nivel de acreditación obtenidos por las carreras, se observa un patrón similar, ya que en su mayoría consideran que estos cambios son responsabilidad del nivel central, siendo las carreras con un nivel de acreditación medio (4-5 años) quienes más asignan responsabilidad a este nivel de la gestión institucional (62,8\% de las carreras de estas categorías).

Respecto a la dimensión Capacidad de Autorregulación, las carreras consideran que los cambios producidos en ésta son más 
bien de responsabilidad del nivel central (59,5\%), observándose una gradiente de mayor a menor responsabilidad en las carreras de categoría $1(68,4 \%)$ a categoría $4(53,4 \%)$. En cambios tales como definición de políticas de docencia y formalización de procesos a través de manuales o guías de procedimientos, las carreras de categoría 1 le atribuyan una mayor responsabilidad al nivel central $(94,1 \%$ en ambos casos) que las de categoría 4 (54,1\% y 45,9\%); estas diferencias son también estadísticamente significativas $(\mathrm{F}=4,241 \mathrm{p}<0,05 ; \mathrm{F}=4,582$ $\mathrm{p}<0,05$, respectivamente). De la misma forma, hay cambios en que las carreras se sienten más responsables, a saber, mecanismos de difusión de reglamentos de carrera en los distintos estamentos $(55,4 \%)$ y comités de autoevaluación $(64,3 \%)$. Independiente del nivel de acreditación de la carrera, todas consideran que muchos de los cambios que se producen en esta dimensión son responsabilidad del nivel central (56\%), observándose patrones similares a los ya descritos en el párrafo anterior.

\section{Estabilidad de los cambios y su relación con los procesos de acreditación conducidos por CNAP}

Un apartado del cuestionario preguntaba a los participantes por la estabilidad de una serie de resultados que podían ser atribuidos al proceso de acreditación conducido por la CNAP, distinguiendo entre disminución, mantención, aumento o mejoría de los mecanismos o componentes organizacionales que experimentaron modificaciones. Todas las carreras, sin importar a qué categoría pertenecieran, señalaron que los resultados enunciados (en el cuestionario) considerados como efectos de la acreditación CNAP en las dimensiones Perfil de Egreso y Condiciones de Operación, se mantenían o aumentaban en el tiempo. En esta última dimensión, las carreras destacaron el aumento en dotación de infraestructura y recursos para la docencia $(60,3 \%)$, mejoría en calidad de la docencia $(64,4 \%)$ y aumento de la dotación de material bibliográfico (72,2\%). Llama la atención que las carreras de la categoría 2 consignaron un aumento significativo en la proporción profesores/alumnos (56,3\%; F=2,727 p<0,05) y en la dotación de docentes jornada completa equivalentes, adecuado a los propósitos de la carrera $(62,5 \% ; F=3,434$ p $<0,05)$, en comparación con las otras categorías, que no destacan a éstos como cambios importantes. 
En la dimensión Capacidad de Autorregulación, se dan algunas diferencias entre categorías; las carreras de las categorías 1 y 3 consideran que los resultados (todos valorados positivamente) producto de la acreditación CNAP aumentaron en un 63,8\%, mientras que sólo un $48,6 \%$ de las carreras de categoría 4 comparte esta percepción.

Al analizar los datos según el nivel de acreditación obtenido por las carreras, se observa un patrón similar al ya descrito. Destaca en la dimensión Capacidad de Autorregulación que las carreras con niveles bajos y medios de acreditación consideran que los resultados que se han producido en la carrera producto de la acreditación CNAP han aumentado (64,9\%). Mientras que menos carreras con un nivel alto de acreditación refieren a cambios que impliquen un aumento (o mejoría) de ciertas condiciones del funcionamiento organizacional en sus unidades académicas. $(40,6 \%)$. Estas diferencias son estadísticamente significativas $(\mathrm{F}=3,416 \mathrm{p}<0,05)$.

\section{Conclusiones}

Si bien se dio cumplimiento a los objetivos del estudio, los resultados deben analizarse con cierta cautela, ya que se presentaron dificultades durante el procedimiento que afectan las condiciones metodológicas del estudio, particularmente en su diseño muestral.

Inicialmente se determinó una cuota mínima aceptable de respuestas por categoría, pero hubo una baja tasa de respuesta de carreras de la categoría 4 (sólo 16\%). Hay distintos elementos que podrían explicar el comportamiento diferencial en la tasa de respuestas a lo largo de las categorías de carreras participantes del estudio. Por un lado, hubo varias afectadas por el terremoto -ocurrido mientras se llevaba a cabo este estudio- en instituciones de regiones que sufrieron los mayores daños en el país, pero también hubo algunas de la Región Metropolitana, sin mayores daños por el sismo, que probablemente tienen un bajo interés por estos temas y fueron resistentes a colaborar pese a las insistencias del equipo de investigación. Estas circunstancias introducen una limitación, que restringe la generalización de los resultados a aquellas unidades que comparten los mismos atributos. 
En relación con los hallazgos, queda de manifiesto la existencia de una relativa homogeneidad en las respuestas de los participantes, tanto en la identificación de cambios como en la importancia que les asignan a los mismos. Estos resultados no sorprenden, dadas las características de las carreras que conformaban el universo. Todas pertenecen a un subgrupo de programas que participaron voluntariamente en procesos de acreditación en un periodo "experimental", antes que hubiera normas que regularan y condicionaran sus resultados. Es así como las carreras de la muestra se sometieron a la evaluación de sus pares, sin otra "moneda de cambio" que los beneficios intrínsecos de todo proceso de autoevaluación y evaluación, con miras al mejoramiento continuo del quehacer académico.

Por otro lado, aunque las carreras presentaron perfiles de respuestas bastante homogéneos, se observaron ciertas diferencias:

- Las carreras de la categoría 4 reconocen una proporción menor de cambios positivos que atribuyen a su participación en procesos de acreditación, de acuerdo a los indicadores contenidos en los reactivos del cuestionario.

- Hay condiciones de operación que no son reconocidas por las carreras de la categoría 4 , aunque las otras sí las señalan entre los cambios como consecuencia de la acreditación. Tal vez, estas carreras presentaban condiciones preexistentes de mejor nivel y más consolidadas, de manera que no se vieron impactadas por los procesos de evaluación externa, como sí lo manifiestan las carreras de las tres primeras categorías.

- Hay factores que convocan mayor acuerdo entre las carreras de distintas categorías y éstas se relacionan con ciertas tareas que se consideran de gestión exclusiva de las disciplinas -relacionadas con la definición del perfil de egreso y el plan de estudios-a diferencia de otras que se consideran de responsabilidad del nivel central de la institución, las que corresponden principalmente a gestiones relacionadas con la autorregulación de la unidad -definición de políticas de docencia y formalización de procesos-.

Por último, un resultado no esperado fue la aplicabilidad de la taxonomía elaborada para clasificar las carreras en este estudio. 
Las distinciones lograron diferenciar las carreras y distribuirlas en un continuo, generando algunos perfiles distintivos entre carreras, por categoría.

Se podría pensar que el continuo sobre el que se distribuyen las carreras en esta taxonomía, probablemente tiene alguna variable "latente" o subyacente, relacionada con los criterios que configuraron las categorías. A modo de hipótesis, probablemente ésta se asocie con procesos de aprendizaje organizacional, los que a su vez dependen de un cierto carácter emprendedor o innovador de las instituciones (Clark, 1998, 2004), en las que puede haber una distribución en elementos estructurales como la diversificación de la base de financiamiento y otros, de orden cultural, como es la convicción de la necesidad de expandir sus actividades, buscar nuevas oportunidades e intervenir en el entorno. Algunas variables específicas que pueden estar en la base de la capacidad de emprendimiento son, de acuerdo a Kuratko et al. (1990), el apoyo administrativo para el emprendimiento, estructura organizacional, riesgo, disponibilidad de tiempo y recursos. Un análisis más a fondo de las características de las carreras que conformaron la base muestral del estudio podría llevarnos a confirmar la presencia de estos factores, los que eventualmente pudieron actuar como facilitadores y modeladores de su participación en procesos de mejoramiento de la calidad de la educación que imparten.

Finalmente, a partir de estos datos, no se pueden hacer afirmaciones acerca de cambios que se producen en carreras que se acreditan obligatoriamente o los que se involucran en estos procesos, estimulados sólo por motivaciones de mercado u otras razones que no se vinculan con la búsqueda genuina de la calidad. Quedan abiertas estas interrogantes para estudios futuros, los que debieran analizar muestras más numerosas y representativas de toda la diversidad de instituciones de educación superior. Se espera que en el mediano plazo, los estudios de esta línea puedan aportar al sistema con el desarrollo de indicadores que permitan la medición del impacto y superen la evaluación indirecta a través de "cuasi indicadores", como son las percepciones y juicios de las partes interesadas. 


\section{Referencias bibliográficas}

Allen, J. y van der Velden, R. (Eds.) (2007) The flexible professional in the knowledge society: General results of the REFLEX project. Disponible en: http://www.educpros.fr/uploads/media/reflex_01.pdf.

Amaral, A. (2010) Impacto del aseguramiento de la calidad en la eficacia formativa. Serie Seminarios Internacionales CNED. ISBN 978-956-7434-26-8. Tomo $13^{\circ}$, abril de 2010 .

Aneca (2007) El profesional flexible en la sociedad del conocimiento. Disponible en: http://www.aneca.es/estudios/docs/InformeejecutivoANECA_ jornadasREFLEXV20.pdf.

Bellingham, L. (2008) Quality Assurance and the Use of Subject Level Reference Points in the UK. Quality in Higher Education, 14 (3), 265-276.

Atria, R. (2006) La gestión de las universidades del Consejo de Rectores. Revista Calidad en la Educación n. ${ }^{\circ}$ 24, pp. 67-116.

Beneitone, P., Esquetini, C., González, J., Marty Maletá, M., Siufi, G., Wagenaar, R. (Eds.) (2007) Reflexiones y perspectivas de la educación superior en América Latina. Informe final proyecto Tuning América Latina 2004-2007. Disponible en: http://tuning.unideusto.org/ tuningal/index.php?option=com_docman\&Itemid=191\&task=view_ category\&catid=22\&order=dmdate_published $\&$ ascdesc $=$ DESC

Briones, G. (1985) Evaluación de Proyectos Sociales. Santiago: PIIE.

Comisión Nacional de Acreditación de Pregrado (2002) Manual de Pares Evaluadores. Ministerio de Educación. Chile.

Clark, B. (1998) The Entrepreneurial University: Demand and Response, Tertiary Education and Management. Vol. 4, n. ${ }^{\circ}$ 1, 5-6.

Clark, B. (2000) The Entrepreneurial University: New Foundations for Collegiality, Autonomy, and Achievement. Higher Education Management. Vol. 13, n. ${ }^{\circ}$ 2. 9-24.

Clark, B. (2004) Delineating the Character of the Entrepreneurial University. Higher Education Policiy. 17, 355-370.

Fernández-Ballesteros, R. (1996) Evaluación de programas. Una guía práctica en ámbitos sociales, educativos y de salud. Madrid: Editorial Síntesis.

Fernández Lamarra, N. (2004) Evaluación y Acreditación de la Educación Superior en la Educación Superior Argentina. En: La Evaluación y la Acreditación en la Educación Superior en América Latina y el Caribe. UNESCO-IESALC, pp. 15-30. Disponible en: http://www.iesalc.unesco. org.ve/estudios/regionales_lat/evalyacredalc.pdf. 
Fernández Lamarra, N. (2007) Educación Superior y calidad en América Latina y Argentina: los procesos de evaluación y acreditación. EDUNTREF, Buenos Aires.

Kuratko, D.; Montagno, R.; \& Hornsby, J. (1990) Developing an Intrapreneurial Assessment Instrument for an Effective Corporate Entrepreneurial Environment. Strategic Management Journal, Vol. 11, 49-58.

Lemaitre M.J. (2005) Aseguramiento de la calidad en Chile: impacto y proyecciones. En Aseguramiento de la calidad: impacto y proyecciones. Santiago de Chile: Serie Seminarios Internacionales, Consejo Superior de Educación.

Mora, J. G. (2004) La necesidad del cambio educativo para la sociedad del conocimiento. Revista Iberoamericana de Educación (35), 13-37. Recuperado el 11 de mayo, 2009, de http:/www.rieoei.org/rie35.htm

Monetta, J.C. y Muñoz, R. (2004) Primera carrera técnica acreditada con la Comisión Nacional de Acreditación de Pregrado. Revista Calidad en la Educación n. ${ }^{\circ} 21$. pp. 207-219.

Navarro, G. (2007) Impacto del proceso de acreditación de carreras en el mejoramiento de la gestión académica. Revista Calidad en la Educación, 26(1), pp. 247-288.

Organización para la Cooperación y el Desarrollo Económico, OECD (2009) La Educación Superior en Chile. Edición en español del Ministerio de Educación de Chile.

Perellon, J. (2007) Analysing Quality Assurance in Higher Education: Proposals for a conceptual framework and methodological implications. En Translation and Transformation, 155-178, Westerheijden et al. (eds.), Quality assurance in higher education: trends in regulation.

Rivera, F., Astudillo, P., Fernández, E. Información y toma de decisiones: oficinas de análisis institucional. Revista Calidad en la Educación - n. ${ }^{\circ}$ 30, julio 2009.

Scharager, J., Cortés, F. y Bravo, T. (2008) Percepción de la Calidad Actual de los Titulados y Graduados de la Educación Superior Chilena. Centro de Medición de la Escuela de Psicología de la Pontificia Universidad Católica de Chile, financiado por Mineduc y BID.

Stensaker, B. (2008) Outcomes of Quality Assurance: A Discussion of Knowledge, Methodology and Validity. Quality in Higher Education, 14 (1), pp. 3-13.

Stufflebeam, D. y Shinkfield, A. (1985) Evaluación sistemática. Guía teórica y práctica. España: Centro de Publicaciones del Ministerio de Educación y Ciencia (M.E.C.) y Ediciones Piados Ibérica, S.A. 
Teichler, U. (2002) Graduate employment and work in Europe: diverse situations and common perceptions. Tertiary Education and Management, 8, 199-216.

Teichler, U. (2007) Does higher education matter? Lessons from a comparative graduate survey. European Journal of Education, 42 (1), pp. 11-34.

Tuning Project (2006) Una introducción a Tuning Educational Structures in Europe. La contribución de las universidades al proceso de Bolonia. Disponible en: http://tuning.unideusto.org/tuningeu/images/stories/template/ General_Brochure_Spanish_version.pdf

Velasco, N., Fernández, R., \& Martínez, Y. (2006) Indicadores y estándares internacionales de calidad universitaria. Revista Calidad en la Educación (25), pp. 17-29.

Zurita, R. (2004) La carrera de psicología en la Universidad de La Frontera: una experiencia de acreditación. Revista Calidad en la Educación n. ${ }^{\circ} 21$, pp. 195-205. 